\title{
Rechtsgeschichte
}

http://www.rg-rechtsgeschichte.de/rg12

Zitiervorschlag: Rechtsgeschichte Rg 12 (2008)

$\operatorname{Rg} 12_{2008}$

$185-188$

http://dx.doi.org/10.12946/rg12/185-188

\section{Benjamin Kram}

Der träumende Jurist 
On the whole one is surprised that the articles (and sections in articles) on Scandinavian legal history have almost no references to literature in Scandinavian languages.

The last major theme of the book concerns the understanding of normative views during the centuries after the fall of the Roman world. There are several ways of dealing with this complex issue, regarding the interpretations of the historical sources and their socio-political contexts on the one hand and contemporary models on law on the other. This perspective is taken rather far by Joachim Rückert who deals with the possible relevance of contemporary discourses on law for the study on pre- and non-state normative structures and practises. Drawing on Luhmann, Hayek and Hart, Rückert discusses a number of topics dealing with these normative structures. Even though Rückert's conceptual analysis due to his distanced relationship to the historical sources in the end says more about the modern legal world than the medieval one, his article is refreshing as it aims more directly at a contemporary legal understanding of the legal world of the early Middle Ages, a perspective lacking in most of the other contributions. And paradoxically that was exactly why the general Nordic scholars read this kind of literature at the turn of the century.

Dag Michalsen

\section{Der träumende Jurist}

\section{Erratum*}

Dass auch die Geisteswissenschaften durchaus in der Lage sein können, Drittmittel nicht unerheblichen Umfangs einzuwerben, zeigt die 2004 fertig gestellte Edition des Traumtraktats von Johannes de Legnano (I320-I383): Sie konnte, wie bereits ein früheres Werk ${ }^{\mathbf{I}}$ über das Leben dieses bedeutenden Kanonisten, dank der finanziellen Förderung durch die Banca Di Legnano in einer Luxusausgabe veröffentlicht werden. Dabei hatte es viele Jahre den Anschein, als entzöge sich ausgerechnet das Sompnium einer kritischen Edition. Zwar hatte Giuseppe Ermini bereits $1940^{2}$ eine Teiledition gewagt, doch scheiterte in den 8 oer Jahren ein erstes, in Oxford begonnenes umfassendes Editionsprojekt am Unfalltod der damals daran arbeitenden Doktorandin. ${ }^{3}$
Johannes berichtet in seinem Sompnium von einer in der Nacht des I 4. Februar I 372 erlebten Traumerfahrung (29I der Edition): Zwei Königinnen seien ihm in diesem Traum erschienen: »et [vidi] (...) duas reginas fulgidissimas: a destris unam habitu regolari decoratam et hec (sic!) canonica sapientia vocabatur; a sinistris alteram habitu seculari honestissimo tamen indutam et haec civilis scientia pingebatur " (7). Kanonistik und Legistik bitten im Traum des Johannes den Papst um Verteidigung gegen das sleere Geschwätz (vaniloquia) der Artisten (pseudo artistae), Theologen und Ärzte, die allesamt den Wissenschaftsstatus beider Rechte verneinen.

Nun ist es also vollbracht: Auf knapp 300 großformatigen Seiten liegt die von Giulietta Voltolina betreute kritische Edition dieses sonderbaren Texts vor, samt einem kenntnisreichen Beitrag über Johannes de Legnano von Maria

\footnotetext{
* Giovanni da Legnano, Somnium. Giorgio D'Ilario, Giovanni da Legnano: le origini (I944); Maria Consiglia de MatTEIS, Diritto e politica nel »Somnium « di Giovanni da Legnano (59-80); Giulietta Voltolina, Somnium. Edizione Critica, Legnano: Banca di Legnano 2004, $293 \mathrm{~S}$.
}

\footnotetext{
I Egidio Gianazza, Giorgio D'Ilario, Vita e Opere di Giovanni da Legnano, Legnano: Banca di Legnano I983.

2 Giuseppe Ermini, Un ignoto trattato »De Principatu « di Giovanni da Legnano, in: Studi di Storia e Diritto in Onore di Carlo Calisse, Milano I940, vol. 3, 42 I-446.

3 G. M. Donovan, K. H. Keen, The 'Somnium of John of Legnano, in: Traditio 37 (I98I) 325-345.
} 
Consiglia de Matteis. Auf den ersten Blick beeindruckt die Edition nicht nur durch die ungewöhnlich großzügige Textanordnung, die das spätgotische Schriftbild, bestehend aus jeweils zwei Kolumnen pro Seite, nachzuempfinden sucht, sondern auch durch die üppige Ausstattung der Edition: In unregelmäßigen Abständen sind ganzseitig farbige Hochglanzreproduktionen aus der Handschriftensammlung Vat. lat. 2639, der eine für die Edition verwendete Handschrift entstammt, in den Editionstext eingefügt. Vervollständigt wird die Edition (ohne Übersetzung) durch zwei Apparate: einen kritischen, der alternative Lesarten zur Leithandschrift wiedergibt, und einen weiteren, der alle Zitate des Haupttextes nachzuweisen sucht.

So weit, so gut. Sobald sich der Leser aber an die detaillierte Textarbeit macht, ist er mit zahlreichen Ungereimtheiten konfrontiert. Zunächst wird er schnell feststellen, dass es sich bei den aufwändigen Reproduktionen nicht etwa um eine Wiedergabe aus derjenigen Handschrift handelt, die der Edition zugrunde lag, sondern um einen völlig anderen Traktat aus der Handschriftensammlung Vat. lat. 2639 (nämlich De Adventu Christi von Johannes de Legnano). Dies wohl nur deshalb, weil dieser Traktat durch ganzseitige Miniaturen sowie prunkvoll gestaltete Initialen eingeleitet wird. Das Sompnium, das sich im hinteren Teil der vatikanischen Prachthandschrift Vat. lat. 2639 befindet, weist außer einigen schlichten, den Text flankierenden gotischen floralen Ornamenten keine farbliche oder bildliche Gestaltung auf. Dabei wäre es eine hervorragende Idee gewesen, den traditionellen kritischen Apparat durch gut lesbare Faksimiles des edierten Texts zu ergänzen und es dem Leser so zu ermöglichen, den Originaltext mit den Vorschlägen der Editorin zu vergleichen. $\mathrm{Zu}$ einer solchen kritischen Arbeit sind die einge- fügten Textreproduktionen aber offensichtlich nicht bestimmt, eher zeugen sie von der antiquarischen Lust am bunten Bildchen: Wie sonst ist zu erklären, dass Folio 7 (2I) vor Folio 6 (32) wiedergegeben ist?

Ein weiteres Rätsel für den Leser sind einige der editorischen Eingriffe in die mittelalterliche Textvorlage. Manchmal erweitert die Editorin Kontraktionen, manchmal nicht, wie hec und haec im obigen Zitat zeigen. Während der lateinische Text (typischerweise) keine Kommasetzung kennt, scheint die Kommasetzungslust der Editorin grenzenlos. Robert B. C. Huygens hat vor nicht allzu langer Zeit dem angehenden Editor mittelalterlicher Texte einige grundlegende Ratschläge gegeben. ${ }^{4}$ Er warnt (54): »Don't scatter commas all over the text, just keep together, what belongs together, both grammatically and logically, instead of breaking up well-balanced phrases. Far from assisting the reader, too many commas only serve to obscure the structure of the phrase and the development of the thought". Giulietta Voltolina scheint Huygens' Warnung vollständig in den Wind geschlagen zu haben. Bei kurzen Sätzen, wie auf S. ro ihrer kritischen Edition, am Ende der rechten Spalte, kann sich der Leser noch selbst behelfen und die falsche Kommasetzung vor dem Accusativus cum Infinitivo ignorieren: "Tradiderunt enim philosophy [,] deum non posse fieri creaturam hominem (...) «. Anders verhält es sich da mit grammatikalischen Strukturen wie dem sich über zwei Spalten hinziehenden zweiten Satz aus dem zweiten Kapitel (Einteilung der Editorin) des Prologs (6): »Nam cum scientiarum omnium dogmata vigilimente revolvo, invenio has duas reginas illustrissimas, canonicam et civilem sapientiam, extimatione multorum oculorum consortis relegatas, solitus sum licet imperitus doctissimus coram iuris et maxime nunc apud reverendissi-

4 Robert B. C. Huygens, Ars

Edendi. A practical introduction to editing medieval Latin texts, Turnhout 2000 . 
mum patrem et dominum meum, dominum Petrum sancte Marie in Transtiberim, presbiterum cardinalem, alme civitatis Bononiae nec non quam plurimum provinciarum Ytalie pro sancta romana ecclesia vicarium generalem, iuris canonici radiis perlustratum, harum exilium ingemiscere dumque hinc inde mutua vice verborum huius rei sermo semel traberetur(,) quia harum sapientiarum foret effectus et que inter eas convenientia nasceretur(,) magno diei spatio alterna disputaione consumpto[,] proxima nocte sequenti casus novitate mirabilis est obortus. " (Die fehlenden Kommas stehen in runden Klammern, das falsche Komma in der eckigen Klammer.)

Ebenso undurchsichtig wie der Editionstext erscheint auch Voltolinas kritischer Apparat. Ohne Auswahl stellt sie einfach alle möglichen und unmöglichen Varianten, die sie in den fünf von ihr verwendeten Handschriften finden konnte, zusammen. Nicht ein einziges Mal hat sie sich die Mühe gemacht, zu entscheiden, welche Variante das Verständnis des Editionstextes erhöhen kann und welche Variante schlicht falsch ist und damit gar nicht erst erwähnt werden sollte. Das hat zur Folge, dass der kritische Apparat unverhältnismäßig aufgeblasen wird und damit jegliche Übersichtlichkeit verloren geht: Die unterstrichene Stelle im obigen Zitat beispielsweise ergibt keinen Sinn. Als alternative Lesart ist aber impariter angegeben - was sich sinnvoller in den Text integrieren ließe. Diese Lesart ist jedoch nicht in den Editionstext aufgenommen worden - warum nicht? Huygens warnt denn auch auf S. 60 seines Büchleins: »The critical apparatus is an essential part of the edition. What it should not contain are all such readings as are irrelevant to the constitution of the text. (...) Don't think half a page of variants permits a better control of your text than only a few lines may. The opposite is true: the more variants, the harder it becomes to use the critical apparatus «.

Bei solchen Mängeln mögen andere - bei weitem leichter $\mathrm{zu}$ behebende - Unzulänglichkeiten in den Hintergrund treten. So ist der Quellenapparat nur insoweit vollständig, als er solche Zitate belegt, deren Herkunft von Johannes de Legnano selbst benannt wurde. Huygens erinnert (I 2) den Editor aber daran, dass »so many phrases, even in the most unbiblical context, owe at least something to the Vulgate, that you should be able to intuitively recognize their unmistakable ring. (...) It is indeed surprising how much the Vulgate has influenced medieval Latin, not only by means of grammatical constructions and throughout direct quotations, but also by it's implicit use as well (...).« Auf S. 9 der kritischen Edition, rechte Spalte, spricht Johannes von dem verlorenen Sohn, vom dem im Evangelium geschrieben steht, dass er »begehrte sich den Bauch zu füllen mit den Träbern, welche die Schweine fraßen $(. .$.$) : »et propterea filius$ ille prodigus in evangelio responditur, qui de siliquis, quas porci manducabant, ventrem implere cupiebat«. Hätte Voltolina an dieser Stelle nicht aufmerksam werden und Lukas I 5:16 identifizieren müssen?

Man hätte dem Traumtraktat des Johannes de Legnano eine verlässlichere Editorin gewünscht. „A bad edition is not necessarily a useless one, but it remains an unreliable one, and just as a thing of beauty is a joy for ever, an unsatisfactory edition is a long lasting annoyance (Huygens, IO).

\section{Gelungen*}

Die zahlreichen Miniaturen des bereits erwähnten Traktats De Adventu Christi, die völlig

\footnotetext{
* Andrea von Hülsen-Esch, Gelehrte im Bild. Repräsentation, Darstellung und Wahrnehmung einer sozialen Gruppe im Mittelalter (Veröffentlichungen des Max-Planck-Instituts für Geschichte 20I), Göttingen: Vandenhoeck \& Ruprecht 2006, 458 S., ISBN 3-525-35 I99-2
} 
willkürlich in die Edition des Sompnium eingefügt worden sind, bilden - auch - den Gegenstand einer weiteren Publikation. Die Kunsthistorikerin Andrea von Hülsen-Esch beschäftigt sich in ihrer Habilitationsschrift eingehend mit der bildlichen Gruppendarstellung spätmittelalterlicher Gelehrter. Von der Kleidung über Accessoires bis hin zum Raum, zur Haltung, Gestik und Interaktion werden die bildlichen Darstellungen von spätmittelalterlichen Gelehrten in die verschiedensten analytischen Perspektiven eingebettet. Von Hülsen-Esch stellt ihren Untersuchungen eine ausführliche Methodendiskussion voran, die die Grenzen, vor allem aber auch die großen Möglichkeiten dieser Forschungsrichtung aufzeigt. Sodann analysiert sie in jeweils eigenen Kapiteln die bildlichen Repräsentationen von Juristen, Ärzten und Artisten. Dabei beschränkt sie sich nicht auf die Buchmalereien, sondern analysiert hinsichtlich der Juristen beispielsweise auch Fresken. Damit soll das ganze Spektrum der bildlichen Repräsentationen des mittelalterlichen Gelehrten in den Blick genommen werden. Unzählige Details dieser Darstellungen werden von der Autorin auf Aussagen über das akademische Selbstverständnis nicht nur im allgemeinen gesellschaftlichen Kontext, sondern auch im Vergleich zu den Vertretern anderer Disziplinen untersucht. Dabei bildet die Analyse der Kleidung der Gelehrten einen besonderen Schwerpunkt. Ein umfangreicher Anhang mit detaillierten farbigen Abbildungen, die die überzeugenden Beobachtungen der Autorin nachvollziehbar machen, schließt das Buch ab.

Bei den Abbildungen von Juristen in der italienischen Buchmalerei entdeckt von HülsenEsch zahlreiche wiederkehrende Darstellungs- muster, deren subtile Aussagen von Rechtshistorikern bisher kaum gewürdigt worden sind. So wird für die beeindruckenden Miniaturen des bereits erwähnten Traktats De Adventu Christi festgestellt, dass "gruppenspezifische hierarchisierende Anordnungen, (...) die zugleich die autoritären Instanzen eindeutig visualisieren «, unter anderem in den »zahlreichen Szenen mit dreieckiger perspektivischer Anordnung der Personen auf eine erhöht sitzende, zentrale Figur hin " verdeutlicht werden, wie es »auch im $\mathrm{Zu}$ sammenhang mit einer Weltgerichtsszene in einer Sammelhandschrift mit kirchenpolitischen Traktaten « der Fall ist (248). Diese Feststellung bezieht sich ausweislich der dazugehörigen Fußnote auf fol. 2v des Traktats De Adventu Christi aus der Handschrift Vat. lat. 2639.

Nach der Lektüre dieses Werkes kann man mit großem Erkenntnisgewinn zur Betrachtung der Abbildungen in der zuerst besprochenen Edition zurückkehren. Plötzlich wird die hohe thematische Dichte der willkürlich in den Traumtraktat eingefügten Illuminationen deutlich: Sie zeigen den Versuch spätmittelalterlicher Gelehrter, sich einen sozialen Stand zu geben und sich auch politisch in der Matrix der gerade entstehenden Wissenschaftsinstitutionen des spätmittelalterlichen Europas zu etablieren. Auch wenn der iconic turn die deutsche Rechtsgeschichte wohl nie gänzlich erreichen wird, böten Forschungsansätze wie der hier besprochene eine hervorragende Gelegenheit, die Reflexion von Rechtshistorikern über den Komplex 'Recht und Bild $\mathrm{zu}$ beflügeln. 